ERRATUM

Eri Nabeshima • Masashi Murakami • Tsutom Hiura

\title{
Effects of herbivory and light conditions on induced defense in Quercus crispula
}

Published online: May 7, 2003

\section{J Plant Res (2001) 114:403-409}

There are errors in Table 2 of the above-mentioned paper.

The corrected table is shown here.

Table 2. Results of repeated-measures ANOVAs on nitrogen content per unit area (N/area), percent nitrogen (N\%), percent carbon (C\%), and carbon nitrogen ratio $(\mathrm{C} / \mathrm{N})$. ANOVA probability values are shown, with F-values in parentheses. Data are shown for herbivory treatment (control and herbivory), light conditions (closed canopy and gap), and sampling times (first and second), respectively $(n=6)$

\begin{tabular}{|c|c|c|c|c|c|}
\hline Factor & df & $\begin{array}{l}\text { N/area } \\
\left(\mathrm{mg} \mathrm{cm}^{-2}\right)\end{array}$ & $\begin{array}{l}\mathrm{N} / \mathrm{mass} \\
(\%)\end{array}$ & $\begin{array}{l}\mathrm{C} / \mathrm{mass} \\
(\%)\end{array}$ & $\mathrm{C} / \mathrm{N}$ \\
\hline Light conditions (L) & 1 & $<0.001(31.58)$ & $0.001(14.33)$ & $0.610(0.27)$ & $<0.001(22.15)$ \\
\hline Sampling time $(\mathrm{S})$ & 1 & $<0.001(75.70)$ & $0.001(14.25)$ & $0.639(0.23)$ & $0.003(11.06)$ \\
\hline $\mathrm{L} \times \mathrm{H}$ & 1 & $0.418(0.68)$ & $0.944(0.01)$ & $0.459(0.57)$ & $0.421(0.67)$ \\
\hline $\mathrm{S} \times \mathrm{L}$ & 1 & $0.086(3.25)$ & $0.007(8.89)$ & $0.748(0.11)$ & $0.017(6.70)$ \\
\hline $\mathrm{S} \times \mathrm{H}$ & 1 & $0.624(0.25)$ & $0.960(0.00)$ & $0.230(1.53)$ & $0.573(0.33)$ \\
\hline $\mathrm{S} \times \mathrm{L} \times \mathrm{H}$ & 1 & $0.781(0.08)$ & $0.538(0.39)$ & $0.810(0.06)$ & $0.313(1.07)$ \\
\hline error & 20 & & & & \\
\hline
\end{tabular}

E. Nabeshima $(\bowtie) \cdot$ M. Murakami · T. Hiura

Tomakomai Research Station, Hokkaido University Forests, Takaoka,

Tomakomai, Hokkaido 053-0035, Japan

Tel. +81-144-33-2171; Fax +81-144-33-2173

e-mail: enabe@exfor.agr.hokudai.ac.jp 